\title{
Flow dichroism in critical colloidal fluids
}

\author{
T. A. J. Lenstra ${ }^{1}$ and J. K. G. Dhont ${ }^{2}$ \\ ${ }^{1}$ Van't Hoff Laboratory for Physical and Colloid Chemistry, Debye Institute, Utrecht University, Padualaan 8, \\ 3584 CH Utrecht, The Netherlands \\ ${ }^{2}$ Forschungszentrum Jülich, IFF, Weiche Materie, D-52425 Jülich, Germany
}

(Received 24 May 2000; revised manuscript received 1 October 2000; published 15 May 2001)

\begin{abstract}
Due to long-range correlations and slow dynamics of concentration fluctuations in the vicinity of the gas-liquid critical point, shear flow is very effective in distorting the microstructure of near-critical fluids. The anisotropic nature of the shear-field renders the microstructure highly anisotropic, leading to dichroism. Experiments on the dichroic behavior can thus be used to test theoretical predictions on microstructural order under shear flow conditions. We performed both static and dynamic dichroism and turbidity measurements on a colloid-polymer mixture, existing of silica spheres (radius $51 \mathrm{~nm}$ ) and polydimethylsiloxane polymer (molar weight $204 \mathrm{~kg} / \mathrm{mol}$ ). Sufficiently far away from the critical point, in the mean-field region, the experimental data are in good agreement with theory. Very close to the critical point, beyond mean field, for which no theory exists yet, an unexpected decrease of dichroism on approach of the critical point is observed. Moreover, we do not observe critical slowing down of shear-induced dichroism, right up to the critical point, in contrast to the turbidity.
\end{abstract}

DOI: 10.1103/PhysRevE.63.061401

PACS number(s): 82.70.Dd, 05.70.Jk, 47.15.-x, 64.60.Ht

\section{INTRODUCTION}

Critical phenomena have been studied for a long time, and the critical behavior of various quantities is well understood. The main interest has been to understand the difference between the values of critical exponents in the mean-field region and very close to the critical point. Very little is known about the effects of external fields on critical behavior and the critical behavior of properties that are induced by external fields. In particular, nonconservative external fields, which cannot be described in terms of a Hamiltonian or a free energy, do not allow for the well-known thermodynamic approaches that led to the current knowledge of critical phenomena. Shear flow is an example of such a nonconservative external field. The critical behavior of systems in such nonconservative external fields must be studied on the basis of kinetic equations. The most fundamental kinetic equation is the equation of motion for the probability density function of phase-space variables. In principle, equations of motion for macroscopic variables can be derived from this fundamental kinetic equation, which then allows for the prediction of their critical behavior. Alternatively, the measurement of the critical behavior of a macroscopic quantity can be used to test a theoretical prediction for the probability density function under shear flow conditions.

On approach of the gas-liquid critical point, interactions become very long ranged. This leads, for example, to the divergence of the turbidity. In addition, the gradient diffusion coefficient tends to zero so that the dynamics of the system severely slows down, often referred to as critical slowing down. Due to the long-ranged spatial correlations and their very slow dynamics, the microstructural order near the gas-liquid critical point is very sensitive to shear flow. For very small shear rates, the turbidity can go down enormously, changing the sample from being white to almost transparent. This change in turbidity is directly related to the change of the microstructure due to the applied shear field.
Moreover, due to critical slowing down, one observes very slow relaxation of the turbidity after cessation of the shear flow.

Due to the anisotropic nature of the microstructure under shear flow, besides a change of the turbidity, dichroism is induced by the shear flow, that is, the turbidity will depend on the polarization state of the incident light. Measurement of shear induced dichroism, resulting from scattering by the anisotropic microstructure, is a sensitive test for the theoretically predicted microstructure under shear flow. This paper presents experimental data on shear induced dichroism as a function of shear rate and the distance from the critical point. As far as we know, this is the first systematic investigation of shear induced dichroism near the gas-liquid critical point. In Ref. [1], a single measurement of dichroism in a near-critical binary fluid is briefly discussed.

The present study is aimed at the understanding of the critical behavior of dichroism in systems under shear flow, on the basis of the solution of the fundamental equation of motion referred to above. The relevant probability density function is the pair-distribution function, the Fourier transform of which is the structure factor. There are a number of theoretical approaches to obtain an expression for the pairdistribution function for systems under shear flow [2-6]. In this paper we concentrate on an expression for the structure factor that is believed to be valid for colloidal systems consisting of spherical particles close to their gas-liquid critical point. This theoretical prediction is a mean-field result [7-9]. No theory exists yet that is valid beyond mean field.

Experimentally, we find that the shear induced dichroism increases on approach of the critical point, in accordance with mean-field predictions, but unexpectedly decreases again on very close approach of the critical point. Moreover, we find no critical slowing down of the relaxation dynamics of dichroism after cessation of shear flow, right up to the critical point. This is in sharp contrast with the turbidity, which diverges on approach of the critical point, and for 


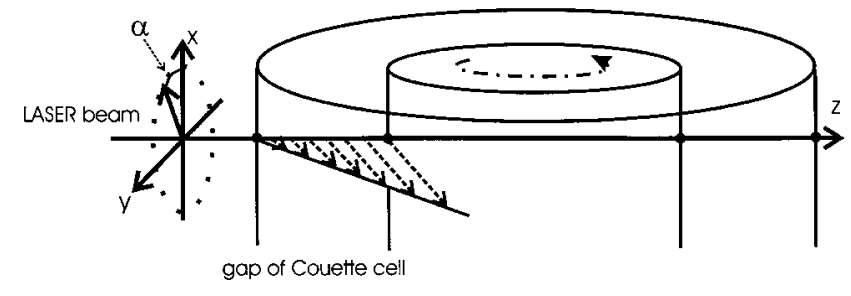

FIG. 1. Geometry used in the theoretical discussion and experimental setup.

which relaxation is severely slowed down.

The colloidal system used is a mixture of spherical colloidal particles and polymers, dissolved in cyclohexane. The added polymer induces attractions, commonly referred to as "depletion attractions" [10-12], which give rise to a gasliquid critical point [13].

This paper is organized as follows. Section II provides the theoretical background that is necessary to understand the data interpretation. In Sec. III the colloidal system is discussed together with the experimental setup that was used to measure dichroism. In Sec. IV the experimental results and comparison with theoretical predictions are presented. Section $\mathrm{V}$ contains some concluding remarks.

\section{THEORETICAL BACKGROUND}

This section contains the necessary theoretical background for the data interpretation that is used in the experimental section. First of all, the relation between the correlation length and the turbidity of a quiescent, unsheared system is discussed. This relation will be used in experiments to determine the distance from the critical point in terms of the correlation length by means of turbidity measurements. Second, the shear distorted stationary structure factor under shear flow is discussed, and the shear induced dichroism will be expressed as a wave-vector integral over this structure factor. This leads to a scaling relation that will be tested experimentally in Sec. IV. Third, the relaxation dynamics of the turbidity and shear induced dichroism, after cessation of the shear flow, is addressed.

The flow that we will consider here is chosen in the $y$ direction, with its gradient in the $z$ direction. That is, the flow velocity is equal to $\mathbf{u}=\Gamma \cdot \mathbf{r}$, where $\Gamma$ is the velocity gradient tensor,

$$
\Gamma=\dot{\gamma}\left(\begin{array}{ccc}
0 & 0 & 0 \\
0 & 0 & 1 \\
0 & 0 & 0
\end{array}\right),
$$

with $\dot{\gamma}$ the shear rate. The direction of the incident laser beam is along the $z$ direction, and the polarization state of the light is specified by the angle $\alpha$ of the electric field with the $x$ axis. This geometry is sketched in Fig. 1 .

\section{A. Relation between scattered intensity and turbidity}

The turbidity measures the loss of intensity as a light beam passes the sample. The sample thickness $I$, the light intensity $I_{s}$ after passing the sample, and the incident intensity $I_{0}$ are connected by Lambert-Beer's law,

$$
I_{s}=I_{0} \exp \{-\tau l\} .
$$

Since the intensity loss is solely due to the scattering of light by the colloidal particles, there is a relation between the turbidity $\tau$ and the integrated scattered intensity, which in turn is proportional to $P(k) S(\mathbf{k})$ with $P(k)$ the formfactor and $S(\mathbf{k})$ the structure factor. This relation reads (see Ref. [7] for the derivation of this equation),

$$
\begin{aligned}
\tau= & \frac{C_{\tau}}{k_{0}^{2}} \int_{0}^{2 \pi} d \varphi \int_{0}^{2 k_{0}} d k k P(k) S(\mathbf{k}) \\
& \times\left[(-\cos \alpha \sin \varphi+\sin \alpha \cos \varphi)^{2}\right. \\
& +(\cos \alpha \cos \varphi+\sin \alpha \sin \varphi)^{2} \\
& \left.\times\left\{1-\left(\frac{k}{k_{0}}\right)^{2}+\frac{1}{4}\left(\frac{k}{k_{0}}\right)^{4}\right\}\right],
\end{aligned}
$$

where the optical constant $C_{\tau}$ is equal to,

$$
C_{\tau}=\frac{k_{0}^{4}}{(4 \pi)^{2}} \bar{\rho} V_{p}^{2}\left|\frac{\bar{\varepsilon}_{p}-\varepsilon_{f}}{\varepsilon_{f}}\right|^{2},
$$

with $k_{0}=2 \pi / \lambda_{\text {wav }}$ ( $\lambda_{\text {wav }}$ is the wavelength of the light in the dispersion), $\bar{\rho}$ the colloidal particle number density, $V_{p}$ the volume of a colloidal particle, $\bar{\varepsilon}_{p}$ the volume averaged dielectric constant of the colloidal particles, and $\varepsilon_{f}$ the dielectric constant of the fluid. The wave-vector $\mathbf{k}$ is equal to $-k_{0}(\sin \theta, \cos \varphi, \sin \theta \sin \varphi, \cos \theta-1)$. The magnitude $k=|\mathbf{k}|$ of the wave vector is equal to $2 k_{0} \sin \theta / 2$, in which $\theta$ is the angle between $\mathbf{k}$ and the $z$ axis. In the integration in Eq. (3) with respect to the spherical coordinates $\varphi$ and $\theta$, the $\theta$ integration is transformed to $k$ integration.

In case the structure factor is isotropic, that is, when $S(\mathbf{k})$ is a function of the magnitude $k=|\mathbf{k}|$ only, the $\varphi$ integration in Eq. (3) can be done analytically, rendering the turbidity independent of $\alpha$. For such isotropic structures there is no polarization dependence of the turbidity. Shear flow renders the structure factor anisotropic, leading to an $\alpha$-dependent turbidity. Shear flow thus induces dichroism through its effect on the microstructure of the suspension of spherical colloidal particles. For nonspherical colloidal particles there is an additional contribution to dichroism that stems from the orientation dependence of the form factor. In the present paper, where spherical colloids are used, such alignment dichroism is absent.

\section{B. Relation between turbidity and correlation length}

The relevance of the relation between the turbidity $\tau$ and the correlation length $\xi$ for the work described in the present paper is that when a dichroism measurement has been done, the correlation length is obtained through a turbidity measurement on the quiescent, nonsheared suspension. Once the relation between the turbidity and the correlation length is 
established, such a turbidity measurement suffices to characterize the distance from the critical point in terms of the correlation length, which is also an important input when comparing with theory.

The structure factor for a nonsheared, equilibrium suspension near the critical point is the Ornstein-Zernike structure factor,

$$
S^{\mathrm{eq}}(k)=\frac{1}{\beta \Sigma} \frac{\xi^{2}}{1+(k \xi)^{2}},
$$

where $\beta=1 / k_{B} T\left(k_{B}\right.$ is the Boltzmann constant and $T$ is the temperature), and $\Sigma$ is a constant related to the Cahn-Hilliard square-gradient coefficient. Furthermore, $\xi$ is the correlation length, which measures the largest distance over which particles are correlated. Equation (4) is a valid expression for the structure factor for small wave vectors, $k<2 \pi / R_{V}$, where $R_{V}$ is the range of the pair-interaction potential. For such small wave vectors, the form factor in Eq. (3) for the turbidity is almost equal to one. Correction terms can be obtained by the Taylor expansion of the form factor of optically homogeneous colloidal spheres,

$$
\begin{aligned}
P(k)= & {\left[3 \frac{\sin (k a)-k a \cos (k a)}{(k a)^{3}}\right]^{2}=1-\frac{1}{5}(k a)^{2}+\frac{3}{175}(k a)^{4} } \\
& +O\left((k a)^{6}\right) .
\end{aligned}
$$

Substitution of Eqs. (4) and (5) into Eq. (3) for the turbidity gives,

$$
\begin{aligned}
\tau^{\mathrm{eq}}(\sigma) \propto \sigma & {\left[\frac{2 \sigma^{2}+2 \sigma+1}{\sigma^{3}} \ln (1+2 \sigma)-2 \frac{(1+\sigma)}{\sigma^{2}}\right.} \\
& +\frac{2}{5} a^{2} k_{0}^{2}\left\{\frac{2 \sigma^{2}+2 \sigma+1}{\sigma^{4}} \ln (1+2 \sigma)\right. \\
& \quad \frac{8}{3} \sigma^{2}+2 \sigma+2 \\
& \left.\left.-\frac{\sigma^{3}}{\sigma^{3}}\right\}+O\left(\left(a k_{0}\right)^{4}\right)\right]
\end{aligned}
$$

where $\sigma=2\left(k_{0} \xi\right)^{2}$. This equation has been derived by Puglielli and Ford [14].

The Ornstein-Zernike structure factor [Eq. (4)] is the contribution to the structure factor due to critical, long-range correlations only, and does not contain the noncritical contributions to the scattered intensity. For the small wave vectors of interest here, this noncritical background is virtually a constant, not only independent of the wave vector, but also independent of the concentration within the vicinity of the critical point. This leads to a noncritical additive, constant contribution to the turbidity in Eq. (6).

Alternatively, the correlation length can be measured from small-angle light-scattering experiments using Eq. (4) for the structure factor. Since the scattered intensity is directly proportional to the structure factor at sufficiently small angles, this equation shows that a plot of $1 / S(k)$ versus $k^{2}$ is a straight line with a slope equal to $\beta \Sigma$ and an intercept equal to $\beta \Sigma \xi^{-2}$. The ratio of the intercept and slope thus equals the squared reciprocal correlation length. As mentioned above, the Ornstein-Zernike structure factor accounts only for the critical contribution to the structure factor. In experimental practice, we shall have to subtract a constant, wave vector independent background intensity.

\section{Critical microstructure under shear flow}

The shear induced turbidity and dichroism can be obtained from Eq. (3) once the structure factor under shear flow is known. Concentration and shear rate-dependent turbidity and dichroism measurements actually serve as a tool to test theoretical predictions for the structure factor under shear flow. Notable theoretical predictions for the shear rate dependence of the structure factor can be found in Refs. [2-6]. Here we discuss a theory that is specialized to colloidal systems close to their gas-liquid critical point [7-9].

Starting point is the Smoluchowski equation, which is the fundamental kinetic equation referred to in the introduction. This is the equation of motion for the probability density function of the position coordinates of the colloidal particles. From this kinetic equation, one obtains the following equation of motion for the structure factor under stationary shear flow (for the geometry sketched in Fig. 1),

$$
\left[\frac{\partial}{\partial t}-\dot{\gamma} k_{2} \frac{\partial}{\partial k_{3}}\right] S(\mathbf{k} ; t \mid \dot{\gamma})=-2 D^{\mathrm{eff}}(k) k^{2}\left[S(\mathbf{k} ; t \mid \dot{\gamma})-S^{\mathrm{eq}}(k)\right] .
$$

Note that in directions where $k_{2}=0$, this equation predicts that there is no effect of the shear flow on the microstructure. Here, $D^{\text {eff }}$ is an effective diffusion coefficient which is equal to,

$$
D^{\mathrm{eff}}(k)=D_{0} \beta\left(\frac{d \Pi}{d \bar{\rho}}+k^{2} \Sigma\right)
$$

with $D_{0}$ the Stokes-Einstein diffusion coefficient of a noninteracting, free colloidal particle, $\Pi$ is the osmotic pressure (of which the derivative with respect to the number density $\bar{\rho}$ is taken), and $\Sigma$ is a constant that is related to the CahnHilliard square gradient coefficient [the same constant occurs in Eq. (4) for the Orstein-Zernike structure factor]. The stationary solution $S(\mathbf{k} \mid \dot{\gamma})$ of this equation of motion is conveniently expressed in terms of the relative structure factor deformation,

$$
\Psi(\mathbf{k} \mid \lambda)=\frac{S(\mathbf{k} \mid \dot{\gamma})-S^{\mathrm{eq}}(k)}{S^{\mathrm{eq}}(k)-1} .
$$

As will become clear later in this subsection, this quantity is well behaved for all wave vectors right up to the critical point. From Eq. (7) one finds, 


$$
\begin{aligned}
\Psi(\mathbf{k} \mid \lambda)= & \frac{1}{\lambda K_{2}} \int_{K_{3}}^{ \pm \infty} d X\left(K^{2}-K_{3}^{2}+X^{2}\right)\left(K_{3}^{2}-X^{2}\right) \\
& \times \exp \left(-\frac{F(\mathbf{K} \mid X)}{\lambda K_{2}}\right)
\end{aligned}
$$

where the dimensionless wave-vector $\mathbf{K}=\mathbf{k} \xi$ is introduced and,

$$
\begin{aligned}
F(\mathbf{K} \mid X)= & \left(X-K_{3}\right)\left(K^{2}-K_{3}^{2}\right)\left(1+K^{2}-K_{3}^{2}\right) \\
& +\frac{1}{3}\left(X^{3}-K_{3}^{3}\right)\left(1+2 K^{2}-2 K_{3}^{2}\right)+\frac{1}{5}\left(X^{3}-K_{3}^{5}\right) .
\end{aligned}
$$

Here, $K_{j}$ is the $j$ th component of the dimensionless wave vector. The upper integration limit in Eq. (10) is $+\infty$ when $\lambda K_{2}>0$ and $-\infty$ when $\lambda K_{2}<0$. Furthermore, $\lambda$ is a dressed Peclet number,

$$
\lambda=\frac{\dot{\gamma} \xi^{2}}{2 D^{\mathrm{eff}}(k=0)}=\frac{1}{\beta \Sigma} \frac{\dot{\gamma} \zeta^{4}}{2 D_{0}} .
$$

In the second equation in Eq. (12) we used that $\xi$ $=\sqrt{\Sigma /[d \Pi / d \bar{\rho}]}$. Note that close to the critical point $d \Pi / d \bar{\rho}$ is small, so that $D^{\text {eff }}$ is small in comparison to the free diffusion coefficient $D_{0}$ for small wave vectors. This reflects the critical slowing down of long wavelength concentration fluctuations.

The dressed Peclet number $\lambda$ in Eq. (12) measures the effect of shear flow on the long-ranged, critical microstructural order. When $\lambda<1$, the critical microstructural order is only slightly affected, while for $\lambda>1$, the effect of shear flow is significant. What is neglected in the derivation of Eq. (10), is distortion of microstructural order extending over distances equal or less than the range $R_{V}$ of the pairinteraction potential. The extent to which such short-ranged microstructural order is affected by shear flow is measured by the so-called bare Peclet number,

$$
\mathrm{Pe}^{0}=\frac{\dot{\gamma} R_{V}^{2}}{2 D_{0}}
$$

Equation (10) is valid whenever $\mathrm{Pe}^{0} \ll 1$. Since $\xi>R_{V}$ and $D^{\text {eff }}<D_{0}$, the dressed Peclet number can be large, also for small bare Peclet numbers. This is due to the fact that large structures are more easily affected by shear flow than small structures, and that long wavelength critical fluctuations are much slower than density fluctuations with small wavelengths $\left(\sim R_{V}\right)$. One can thus distinguish three shear rate regimes,

$$
\begin{gathered}
\lambda<1 ; \operatorname{Pe}^{0} \ll 1 \quad \text { weak shear flow, } \\
\lambda>1 ; \operatorname{Pe}^{0} \ll 1 \quad \text { strong shear flow, } \\
\lambda>1 ; \operatorname{Pe}^{0} k 1 \quad \text { very strong shear flow. }
\end{gathered}
$$

Equation (10) for the structure factor distortion is valid in the weak and strong shear regime, but not in the very strong shear regime.
The result in Eq. (10) is valid only in the mean-field region around the critical point. This is due to a linearization of equations of motion with respect to the total-correlation function $h(r)$ for large distances $r$ between two colloidal particles. In fact, terms $\sim h^{2}$ are neglected against the term $(\beta d \Pi / d \bar{\rho}) h$ for large distances $r$. This is only a valid procedure when $\beta d \Pi / d \bar{\rho}$ is not extremely small, that is, when the distance to the critical point is not too small. The above predictions are therefore only valid in the mean-field region.

In the derivation of Eq. (10), hydrodynamic interactions between colloidal particles have been neglected. Furthermore, a closure relation has been used in order to express the three-particle correlation function in terms of the paircorrelation function. This approximate closure relation is reminiscent of the classic super-position closure relation.

It can be shown rigorously, that the displacement of the critical point for colloidal systems is proportional to $\left(\mathrm{Pe}^{0}\right)^{1 / \gamma}$, where $\gamma=1.23$ is the critical exponent of the compressibility of the quiescent system [15]. The displacement of the critical point for spherical colloids is related to the distortion of the pair-correlation function for short distances, smaller than the range of the pair-interaction potential, and is therefore a function of the bare Peclet number $\mathrm{Pe}^{0}$. For the experiments described here, $\mathrm{Pe}^{0} \leqslant 0.08$, so that the displacement of the critical point is not important for the present work.

Numerical results for the relative structure factor distortion and the structure factor itself are plotted in Fig. 2. In some directions there is enhancement of the structure, whereas in other directions, destruction of the structure is observed, as can be seen from the plots of $\Psi$. This can be understood by decomposing a simple shear flow in its elongational and rotational contributions. The elongational contribution tends to enhance structure along the $y=-z$ direction, and diminishes structure in the direction where $y=z$. These directions correspond to the directions $k_{2}=-k_{3}$ and $k_{2}=k_{3}$, respectively. Note that $\Psi$ is found to be finite right up to the critical point, which is the reason for introducing this quantity.

\section{Shear induced dichroism}

The change of the turbidity on applying a shear flow follows from Eqs. (3), (4), (9), and (10). Transforming to the dimensionless $K=k \xi$ variable, and disregarding the small $\left(K / K_{0}\right)^{4}$ terms in Eq. (3), the turbidity, relative to the turbidity for $\alpha=\pi / 2$, is found to be equal to,

$$
\begin{aligned}
\Delta \tau(\dot{\gamma} \mid \alpha) \equiv & \tau(\dot{\gamma} \mid \alpha)-\tau\left(\dot{\gamma} \mid \alpha=\frac{\pi}{2}\right) \\
= & -\frac{C_{\tau}}{\left(K_{0} R_{V}\right)^{4}} \frac{1}{\left(\beta \Sigma / R_{V}^{2}\right)^{3 / 2}} \sqrt{\mathrm{Pe}^{0}(\dot{\gamma})} \\
& \times\left[\cos ^{2}(\alpha) D_{\mathrm{cc}}(\lambda)+2 \sin (\alpha) \cos (\alpha) D_{\mathrm{sc}}(\lambda)\right]
\end{aligned}
$$

where the scaling functions $D_{\mathrm{sc}}$ and $D_{\mathrm{cc}}$ are given by, 

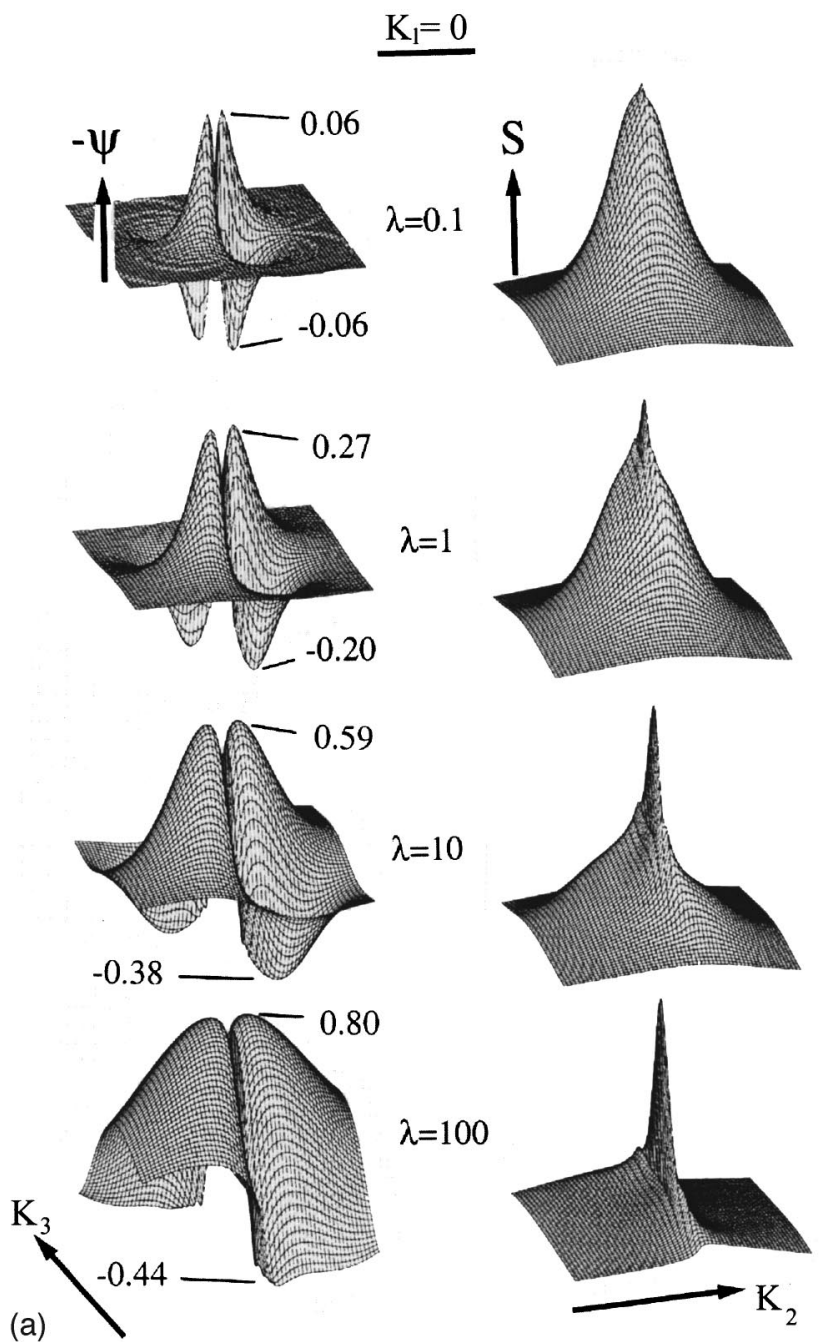
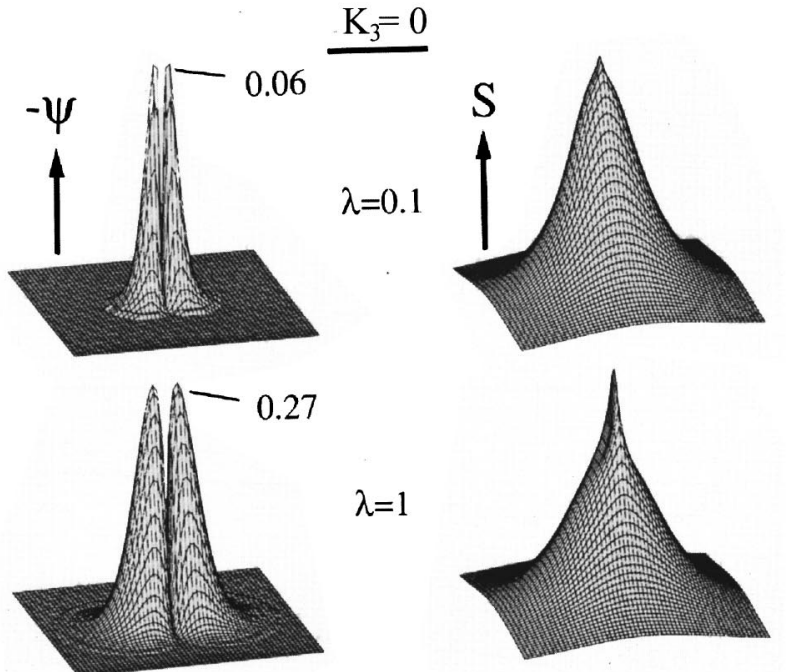

(b)
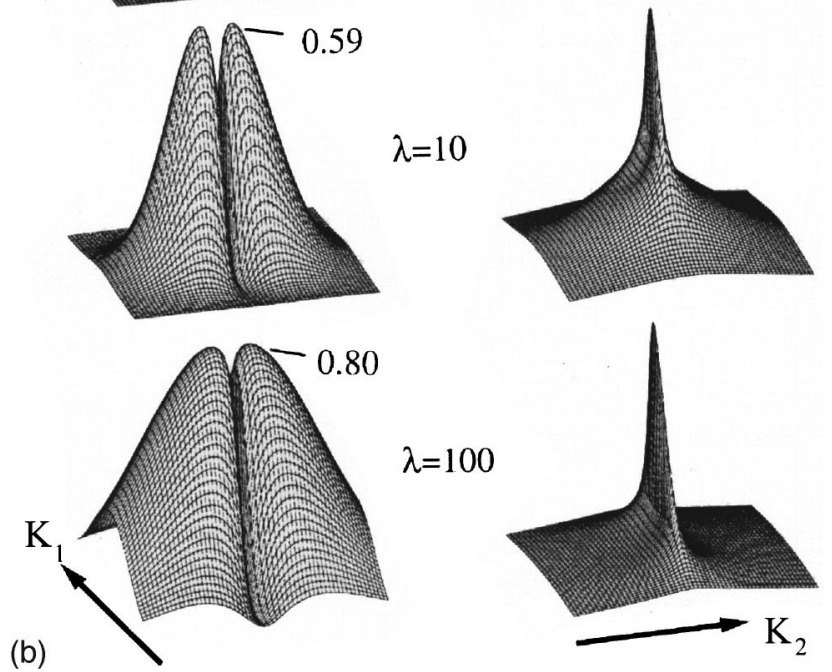

FIG. 2. Plots of (minus) the relative structure factor distortion $\Psi(\mathbf{k} \mid \lambda)$ and the structure factor $S(\mathbf{k} \mid \dot{\gamma})$, at $K_{1}=0$ (a) and $K_{3}=0$, (b) for various values of $\lambda$ as indicated in the figure. $K_{1}, K_{2}$, and $K_{3}$ values range from -3 to +3 . The minimum and maximum values of $\Psi$ are indicated in the figure.

$$
\begin{aligned}
D_{\mathrm{cc}}(\lambda)= & \frac{1}{\sqrt{\lambda}} \int_{0}^{2 \pi} d \varphi \int_{0}^{\infty} d K \frac{K^{3}}{1+K^{2}} \Psi^{*}(K, \varphi \mid \lambda) \\
& \times\left(\cos ^{2} \varphi-\sin ^{2} \varphi\right)
\end{aligned}
$$

and,

$$
\begin{aligned}
D_{\mathrm{sc}}(\lambda)= & \frac{1}{\sqrt{\lambda}} \int_{0}^{2 \pi} d \varphi \int_{0}^{\infty} d K \frac{K^{3}}{1+K^{2}} \Psi^{*}(K, \varphi \mid \lambda) \\
& \times \sin (\varphi) \cos (\varphi) .
\end{aligned}
$$

The upper integration limit $2 k_{0}$ in Eq. (3) is replaced here by $\infty$. This can be done for systems under shear flow, where the structure factor distortion exhibits a peak at small values of $K$, and tends to 0 for large wave vectors much faster than $1 / K^{2}$ (except in directions where $K_{2} \approx 0$ ) as can be seen from the plots of $S(\mathbf{k} \mid \dot{\gamma})$ in Fig. 2. In fact, numerical evaluation of the integrals in Eqs. (15) and (16) shows that the integrals converge to within a fraction of $1 \%$ for an upper integration limit of typically $K \approx 5$. For the same reason, $\Psi$ is replaced here by $\Psi^{*}$, which is equal to $\Psi$ with the angle $\theta$ of $\mathbf{k}$ with the $z$ axis taken into account to leading order, that is,

$$
\Psi^{*}(K, \varphi \mid \lambda)=\Psi(\mathbf{K}=K(\cos \varphi, \sin \varphi, 0) \mid \lambda) .
$$

Numerical evaluation of the scaling functions reveals that for the present geometry (see Fig. 1) $D_{\text {sc }}=0$, to within numerical errors. Numerical results for the scaling functions are plotted in Fig. 3. Here, the curves labeled with a "1" refer to the geometry where the direction of the beam is along the vorticity direction, while ' 2 '" refers to the geometry sketched in Fig. 1. The reason for division by $\sqrt{\lambda}$ in Eqs. (15) and (16) is that numerical evaluation of the scaling functions indicates that these now tend to a constant for large dressed Peclet numbers $\lambda$.

In the weak shear limit, where $\lambda<1$, for the geometry " 2 ', of interest here, $D_{\text {cc }}$ can be found by substitution of a regular expansion of the structure factor with respect to $\lambda$. To this end the expansion, 

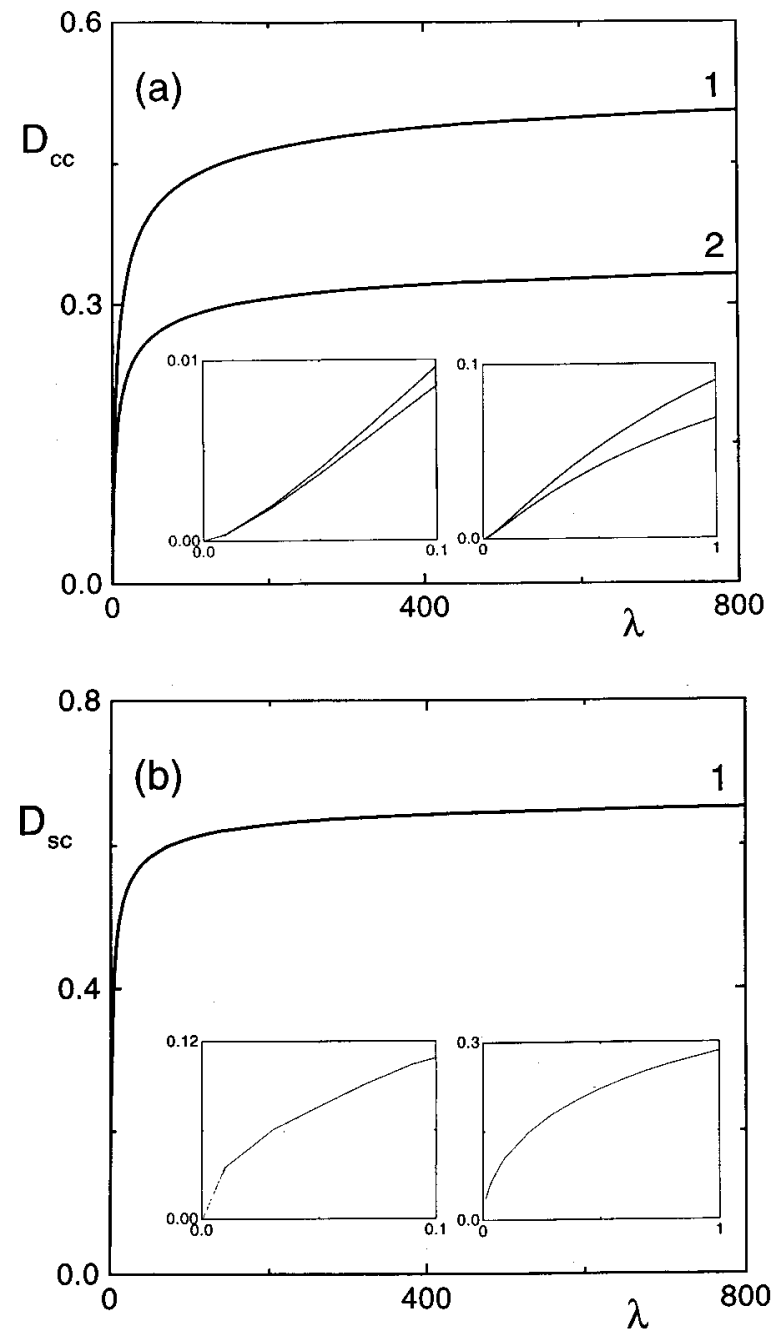

FIG. 3. Numerical results for the scaling functions $D_{c c}$ (a) and $D_{s c}$ (b). The insets show the behavior for small values of $\lambda$, which confirms the analytically predicted behavior as discussed in the main text, below Eq. (18).

$$
S(\mathbf{K} \mid \dot{\gamma})=S^{\mathrm{eq}}(K)+\lambda S^{(1)}(\mathbf{K})+\lambda^{2} S^{(2)}(\mathbf{K})+\ldots,
$$

is substituted into the stationary form of Eq. (7). Equating terms of equal powers in $\lambda$ reveals that, for the geometry sketched in Fig. 1, $S^{(1)} \sim \sin \varphi$ and $S^{(2)} \sim \cos ^{2} \varphi$. The $\varphi$ integration in Eq. (15) for $D_{\text {cc }}$ renders the linear term in $\lambda$ equal to 0 , so that, $D_{\mathrm{cc}} \sim \lambda^{3 / 2}$. Dichroism thus varies like $\dot{\gamma}^{2}$ for small dressed Peclet numbers. The shear induced dichroism in the present geometry is thus inherently nonlinear. The small shear rate behavior of $D_{\mathrm{cc}}$ is plotted in the inset of Fig. 3(a). The function $D_{\text {sc }}$, relevant for the geometry ' 1 ,', varies like $\lambda^{1 / 2}$ for small $\lambda$. In the " 1 ", geometry, there is thus a nonzero linear response. Note the very small range of $\lambda$ 's in Fig. 3 where the leading $\lambda$ dependence is dominant.

The regular expansion Eq. (18) is invalid in a region in wave-vector space around $\mathbf{k}=\mathbf{0}$ of width $\sim \sqrt{\lambda}$, even for small values of $\lambda$. The reason is that Eq. (7) is singularly perturbed by the shear flow contribution. In this so-called (mathematical) boundary layer at $\mathbf{k}=\mathbf{0}$ there is never linear shear flow response of the structure factor. The regular ex- pansion Eq. (18) does make sense, however, when used in integrals like in Eqs. (15) and (16) for the scaling functions, since the width of the boundary layer vanishes for small shear rates, and therefore contributes only little to the value of the integral. In addition, the integrand in Eqs. (15) and (16) vanishes for zero wave vectors, so that the error that is made by using the regular expansion is further diminished.

The maximum variation of the turbidity on variation of $\alpha$ multiplied by $\lambda_{\text {wav }} / 2 \pi$ defines the dichroism $\Delta n^{\prime \prime}$. From Eq. (14), we thus finally find the following expression for the shear induced dichroism for the geometry ' 2 ', near the gasliquid critical point,

$$
\Delta n^{\prime \prime}=\frac{C_{\tau}}{\left(k_{0} R_{V}\right)^{4}} \frac{1}{\left(\beta \Sigma / R_{V}\right)^{3 / 2}} \frac{\sqrt{\mathrm{Pe}^{0}(\dot{\gamma})}}{k_{0}} D_{\mathrm{cc}}(\lambda)
$$

This result predicts scaling in the sense that dichroism depends on the shear rate and the distance from the critical point only through the dressed Peclet number $\lambda$, except for the trivial prefactor $\sqrt{\mathrm{Pe}^{0}(\dot{\gamma})}$. This scaling implies that experimental data for $\Delta n^{\prime \prime} / \sqrt{\dot{\gamma}}$ taken at various shear rates and distances from the critical point should collapse onto a single master curve when plotted versus $\dot{\gamma} \xi^{4}$, the form of which master curve is given by the scaling function in Fig. 3(a) (geometry ' 2 '”).

\section{E. Time-dependent turbidity and dichroism}

Consider an experiment where a stationary shear flow is suddenly switched off, at time $t=0$, say. The solution of Eq. (7) for this situation is a single exponential function of time,

$S(\mathbf{K} ; t \mid \dot{\gamma})=\left[S^{\mathrm{eq}}(K)-1\right] \Psi(\mathbf{K} \mid \lambda) \exp \left(-K^{2}\left[1+K^{2}\right] \frac{\dot{\gamma} t}{\lambda}\right)$.

where the structure factor $S(\mathbf{k} \mid \dot{\gamma})$ in Eq. (9) for $\Psi$ is now the structure factor in the stationary state, before cessation of the flow. The dimensionless variables as defined in subsection $\mathrm{C}$ are introduced here. The time dependent behavior of the turbidity is immediately found by substitution of Eq. (20) into Eq. (3), neglecting terms of order $\left(K / K_{0}\right)^{2}$,

$$
\begin{aligned}
\tau(\dot{\gamma} \mid t)-\tau^{\mathrm{eq}}= & -\frac{C_{\tau}}{\left(k_{0} R_{V}\right)^{4}} \frac{1}{\left(\beta \Sigma / R_{V}^{2}\right)^{3 / 2}} \\
& \times \int_{0}^{2 \pi} d \varphi \int_{0}^{\infty} d K \frac{K}{1+K^{2}} \Psi^{*}(K, \varphi \mid \lambda) \\
& \times \exp \left[-K^{2}\left(1+K^{2}\right) \frac{\dot{\gamma} t}{\lambda}\right]
\end{aligned}
$$

Similarly, as in subsection D, the polarization dependence of the turbidity is given by, 


$$
\begin{aligned}
\Delta \tau(\dot{\gamma}|\alpha| t) \equiv & \tau(\dot{\gamma}|\alpha| t)-\tau\left(\dot{\gamma}\left|\alpha=\frac{\pi}{2}\right| t\right) \\
= & -\frac{C_{\tau}}{\left(k_{0} R_{V}\right)^{4}} \frac{1}{\left(\beta \Sigma / R_{V}^{2}\right)^{3 / 2}} \sqrt{\mathrm{Pe}^{0}(\dot{\gamma})} \\
& \times\left[\cos ^{2}(\alpha) D_{\mathrm{cc}}(\lambda \mid \dot{\gamma} t)\right. \\
& \left.+2 \sin (\alpha) \cos (\alpha) D_{\mathrm{sc}}(\lambda \mid \dot{\gamma} t)\right],
\end{aligned}
$$

where

$$
\begin{aligned}
D_{\mathrm{cc}}(\lambda \mid \dot{\gamma} t)= & \frac{1}{\sqrt{\lambda}} \int_{0}^{2 \pi} d \varphi \int_{0}^{\infty} d K \frac{K^{3}}{1+K^{2}}\left(\cos ^{2} \varphi-\sin ^{2} \varphi\right) \\
& \times \Psi^{*}(K, \varphi \mid \lambda) \exp \left(-K^{2}\left[1+K^{2}\right] \frac{\dot{\gamma} t}{\lambda}\right)
\end{aligned}
$$

and,

$$
\begin{aligned}
D_{\mathrm{sc}}(\lambda \mid \dot{\gamma} t)= & \frac{1}{\sqrt{\lambda}} \int_{0}^{2 \pi} d \varphi \int_{0}^{\infty} d K \frac{K^{3}}{1+K^{2}} \sin (\varphi) \cos (\varphi) \\
& \times \Psi^{*}(K, \varphi \mid \lambda) \exp \left(-K^{2}\left[1+k^{2}\right] \frac{\dot{\gamma} t}{\lambda}\right) .
\end{aligned}
$$

Numerical evaluation of Eqs. (23) and (24) leads to a pronounced difference in relaxation times for the turbidity and dichroism after cessation of the shear flow. Dichroism relaxes fast in comparison to the turbidity. Formally, this is due to the factor $K^{3}$ in the integrand in Eqs. (23) and (24) for the dichroism, as compared to the factor $K$ in Eq. (21) for the turbidity. The dynamics of dichroism is connected to larger wave vectors as compared to the turbidity. The dynamics of concentration fluctuations pertaining to these larger wave vectors are fast compared to small wave vectors.

\section{EXPERIMENT}

\section{A. Colloidal system}

The system used in this study consists of colloidal silica particles grafted with stearyl alcohol. The solvent is cyclohexane. Polydimethylsiloxane (PDMS) is added to induce depletion attractions between the colloidal particles that give rise to a gas-liquid critical point. The silica particles were synthesized by the method of Stöber [16]. The spheres have an average diameter of $102 \mathrm{~nm}$, as determined by dynamic light scattering. The polydispersity, determined by transmission electron microscopy, was found to be around $16 \%$. The specific mass $\rho$ of the silica particles, that relates the volume fraction $\Phi$ to the mass concentration $c$ as $\Phi=c / \rho$, was determined by Ubbelohde measurements, using Einstein's formula $\eta_{r}=1+2.5 \Phi$, which relates the shear viscosity $\eta_{r}$ to the volume fraction $\Phi$ of spheres. The specific mass $\rho$ was found to be $1.863 \mathrm{~g} / \mathrm{ml}$. The polymer PDMS that we used has a molar weight of $204 \mathrm{~kg} / \mathrm{mol}$. When dissolved in cyclohexane, the spherical coils have a radius of gyration of around $26 \mathrm{~nm}\left(\right.$ at $\left.25^{\circ} \mathrm{C}\right)$.

To determine the phase diagram, a number of samples with various, fixed PDMS to silica concentration ratios were

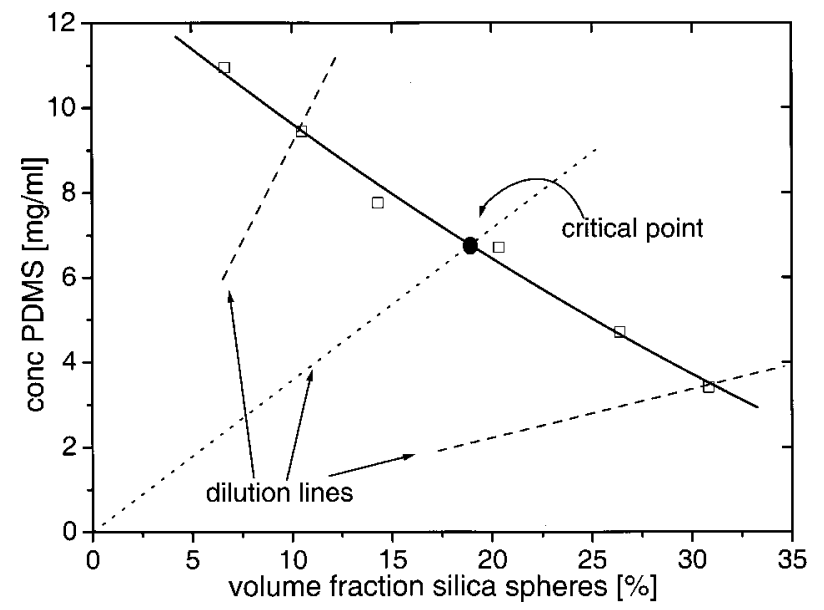

FIG. 4. Experimental phase diagram (polymer to colloid diameter ratio 0.51 ). The binodal points $\square$ are obtained by visual observation.

prepared. By adding or evaporating solvent, we moved along so-called dilution lines in the phase diagram, as depicted in Fig. 4. The binodal was found by visual inspection of the samples for the formation of an interface and the time it takes before an interface could be seen. Close to the critical point, phase separation could take a few hours due to the fact that the density difference between the gas and liquid phases becomes very small. The experimentally determined phase diagram is shown in Fig. 4. The critical point was found by locating the point on the binodal where, after phase separation, the volumes of the two phases are equal. Close to the critical point, phase separation occurred after about two hours. All measurements are performed on a sample on the dilution line that intersects the critical point. The distance from the critical point is varied by gently evaporating or adding solvent.

\section{B. EXPERIMENTAL SETUPS}

The important parameter that characterizes the distance from the critical point, which is also an important input when comparing with theory, is the correlation length. When performing a dichroism experiment, one could measure the concentration of the sample, and from that, derive the correlation length, after the relation between the correlation length and the concentration has been established independently. The relevant differences in concentration, however, are so small that the determination of the concentration would be far too inaccurate. Before and after each dichroism experiment, we therefore performed a turbidity measurement on the quiescent, unsheared sample, and determined the correlation length from the turbidity. To this end, we first have to establish the relation between the turbidity and the correlation length. The experimental setup for this purpose is sketched in Fig. 5. The laser beam first passes two polaroid's in order to be able to adjust the intensity of the beam. A nonpolarizing beam splitter is used to determine the intensity of the incident light with detector 1 . The sample is immersed in a thermostating optical matching bath, to prevent scattering from optical imperfections of the cuvette. The scattered light 


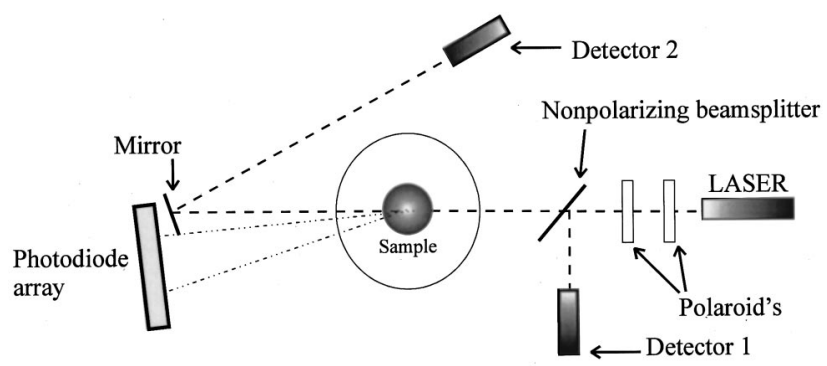

FIG. 5. Experimental setup for the determination of the relation between the turbidity and the correlation length.

intensity is measured by means of a photodiode array camera that is positioned in the focal plane of the optical bath. The scattering angle range is $2-6$ degrees. The intensity of the beam that passed the sample is reflected by a mirror to detector 2. A circular pinhole with a diameter of $0.3 \mathrm{~mm}$ has been used to prevent detection of scattered light by detector 2 . The ratio of the intensities of the detectors 1 and 2 is then recorded for an experiment with the colloidal sample and only solvent, respectively. These two intensity ratios determine the turbidity of the colloidal sample through LambertBeer's law Eq. (2). The path length of the cuvettes is typically $0.2 \mathrm{~cm}$.

The dichroism setup is based on the design by Fuller $[17,18]$. The setup was adjusted to be able to also measure the turbidity of the system, for reasons discussed above. In our setup, the rotating $1 / 2$ waveplatelet is mounted on a dentist drill, which accomplishes a rotational speed of about 4 $\mathrm{kHz}$. The shear cell is a homemade optical couette cell with a gapwidth of $2.47 \mathrm{~mm}$, which is placed in a thermostating, optically matching bath.

Before an actual dichroism experiment was started, the colloid-polymer mixture was left to equilibrate for at least 30 min in the thermostated, optical bath. The turbidity is measured before and after each dichroism experiment in order to verify that evaporation of solvent during the experiment is insignificant. It turned out that evaporation is insignificant over periods of several hours.

\section{EXPERIMENTAL RESULTS AND DISCUSSION}

\section{A. Correlation length dependence of turbidity}

Figure 6 shows the reciprocal intensity versus the wavevector $k$ squared, for a sample with the critical colloid to polymer ratio, for various distances from the critical point (see the dilution line in Fig. 4). The solid lines in this figure are curves fitted to Eq. (4), where the noncritical background contribution to the intensity is used as an additional fitting parameter. Relatively far from the critical point, the curves are virtually straight lines, but closer to the critical point, curves deviate from a straight line. The reason for this is the large variation of the relative contribution of the noncritical background intensity: for small wave vectors, where the critical scattered intensity is very large, the relative contribution of the background intensity is much smaller than for the larger wave vectors. Further away from the critical point, the relative background contribution becomes wavevector inde-

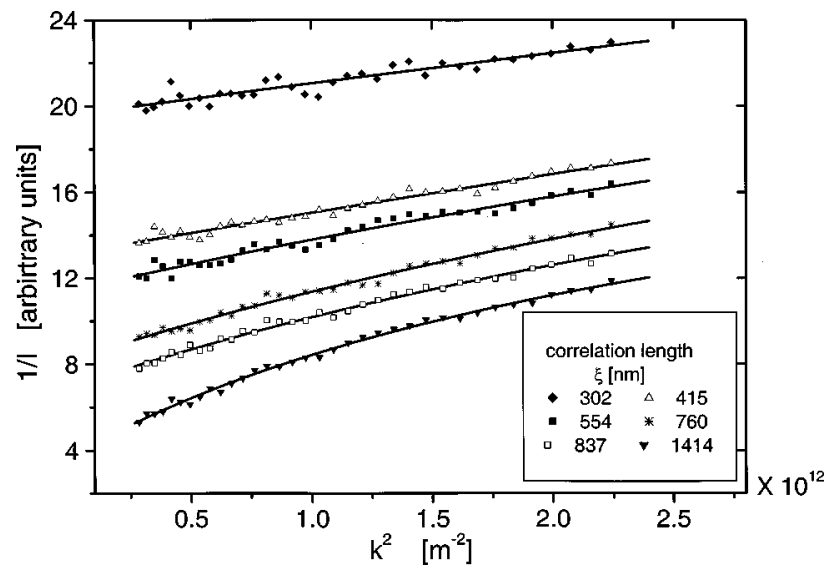

FIG. 6. Reciprocal scattered intensity versus $k^{2}$.

pendent and renders Orstein-Zernike plots linear. Except for the largest correlation lengths shown in Fig. 6, the curves have the same slope. The slope is proportional to $\beta \Sigma$ so that this parameter is seen to be well behaved near the critical point, as expected.

We found that on approach of the critical point, the time needed to render the scattered intensity time independent increases. This is a manifestation of the critical slowing down of the dynamics of concentration fluctuations. For very large correlation lengths (more than about $2500 \mathrm{~nm}$ ) we observed a decrease of the measured correlation length over longer periods of time. This is illustrated in Fig. 7. Right after homogenization of the sample, a fit to the Ornstein-Zernike structure factor, including the noncritical background contribution, yields a correlation length of $424 \mathrm{~nm}$. A measurement after some hours yields a correlation length of 5011 $\mathrm{nm}$. This value then decreases over a period of $8 \mathrm{~h}$ to 1375 $\mathrm{nm}$. The reason for this decrease in correlation length is probably that density inhomogeneities are so long lived, as a result of severe critical slowing down, that sedimentation occurs. The system then develops large scale concentration gradients. The concentration of the part of the system from which scattered intensities are measured differs from the overall concentration, giving rise to a smaller measured cor-

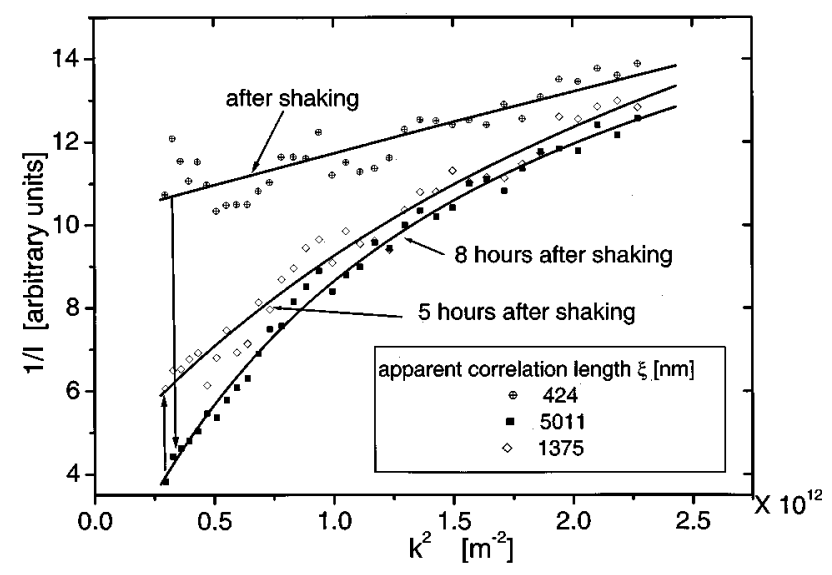

FIG. 7. Time dependence of the reciprocal scattered intensity as a function of $k^{2}$. 


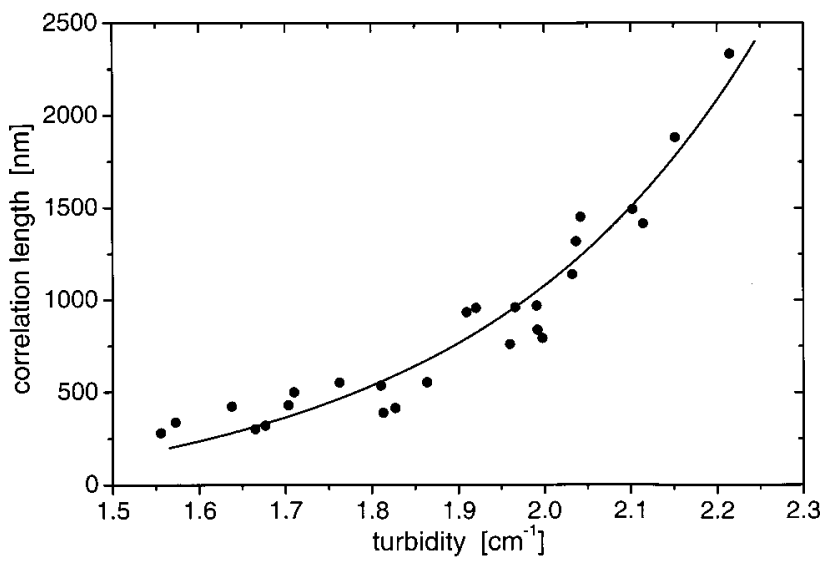

FIG. 8. Relation between the correlation length and the turbidity.

relation length. Measurements are therefore only reliable in case the correlation length is smaller than about $2500 \mathrm{~nm}$.

Figure 8 shows the correlation length versus the turbidity. The smallest correlation length of $250 \mathrm{~nm}$ is about twice as large as the range of the pair-interaction potential, which is equal to the sum of the diameters of the colloidal particles and the polymer diameter of gyration $(\sim 150 \mathrm{~nm})$. The solid curve in Fig. 8 is a fit to Eq. (6). The solid curve will be used to determine the correlation length from turbidity measurements.

\section{B. Dichroism under stationary shear flow}

Measurements were done on a sample with the critical concentration ratio of colloid to polymer. The distance to the critical point is varied by the evaporation or the addition of solvent. The actual distance to the critical point in terms of the correlation length $\xi$ is obtained from transmission measurements and Fig. 8. A transmission measurement was done before and after the dichroism measurements were performed, in order to ensure that no significant evaporation occurred during the dichroism measurement.

Dichroism measurement results in the shear rate range of 0.1 to $34.7 \mathrm{~s}^{-1}$ are plotted in Fig. 9 for various correlation lengths. The lowest curve (dashed line) is the dichroism for a sample with silica particles only, without polymer (PDMS). This curve relates to noncritical contributions to the dichroism. As can be seen, these noncritical contributions are negligibly small as compared to critical contributions.

As can be seen from this figure, dichroism increases with an increasing correlation length up to a certain length after which dichroism decreases. This is made more explicit in Fig. 10, where the dichroism at the maximum applied shear rate $\left(34.7 \mathrm{~s}^{-1}\right)$ is plotted against the correlation length. The mean-field theory described in section II.D predicts a monotonous increase of the dichroism with the correlation length. We therefore conclude that the cross-over from mean field to non-mean field behavior occurs at a correlation length of about $750 \mathrm{~nm}$.

When comparing to the theory described in Sec. II D, we therefore have to restrict to correlation lengths less than 750 $\mathrm{nm}$. Note that since, in Fig. 10, the shear rate is a constant, the location of the critical point is fixed. The decrease of

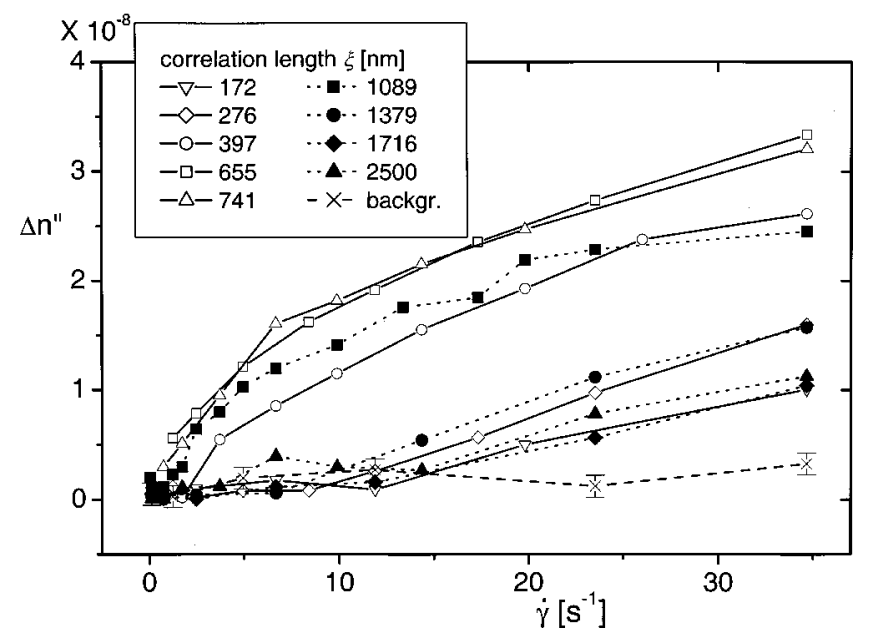

FIG. 9. Dichroism as a function of the shear rate for various correlation lengths, as indicated in the figure. The lowest curve is the dichroism of the sample without polymer.

dichroism can therefore not be attributed to a shear induced shift of the critical point (see also the discussion at the end of in Sec. II C).

\section{Dichroism in the mean field region}

The shear rate dependence of dichroism within the meanfield region is plotted in the insert in Fig. 11. Within the mean-field region, dichroism is monotonously increasing with increasing shear rate. The experiments for small correlation lengths $(<300 \mathrm{~nm})$ suggest that the slope of the dichroism against the shear rate is 0 for small shear rates. This is in accordance with the theoretical result, $\Delta n^{\prime \prime}-\dot{\gamma}^{2}$ for small shear rates.

The theoretical result Eq. (9) implies that $\Delta n^{\prime \prime} / \sqrt{\dot{\gamma}}$ is a function of the shear rate and the correlation length only through the combination $\dot{\gamma} \xi^{4}$. Hence, in a plot of $\Delta n^{\prime \prime} / \sqrt{\dot{\gamma}}$

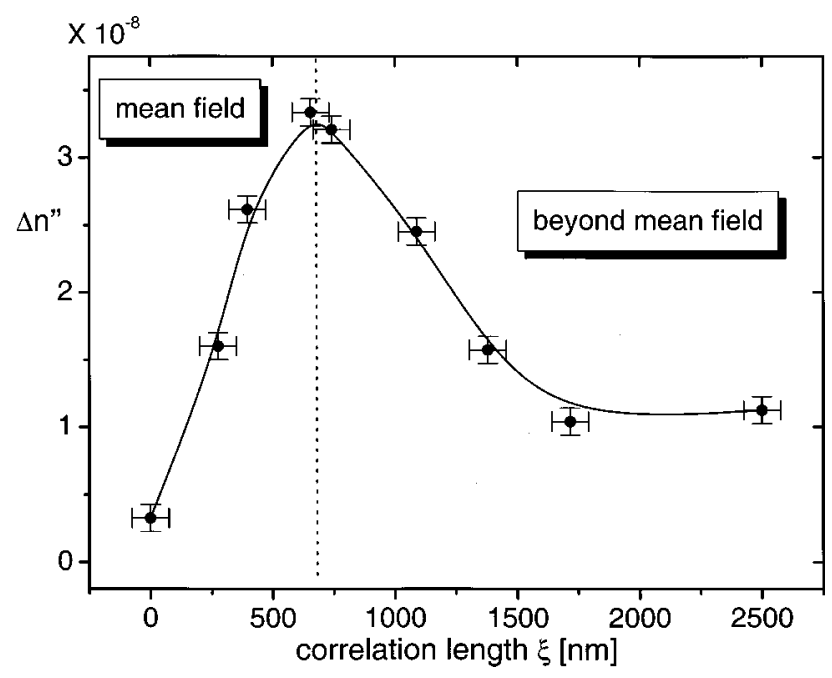

FIG. 10. Dichroism at $\dot{\gamma}=34.7 \mathrm{~s}^{-1}$ versus correlation length. The vertical dotted line indicates where the crossover from meanfield to nonmean-field behavior occurs. 


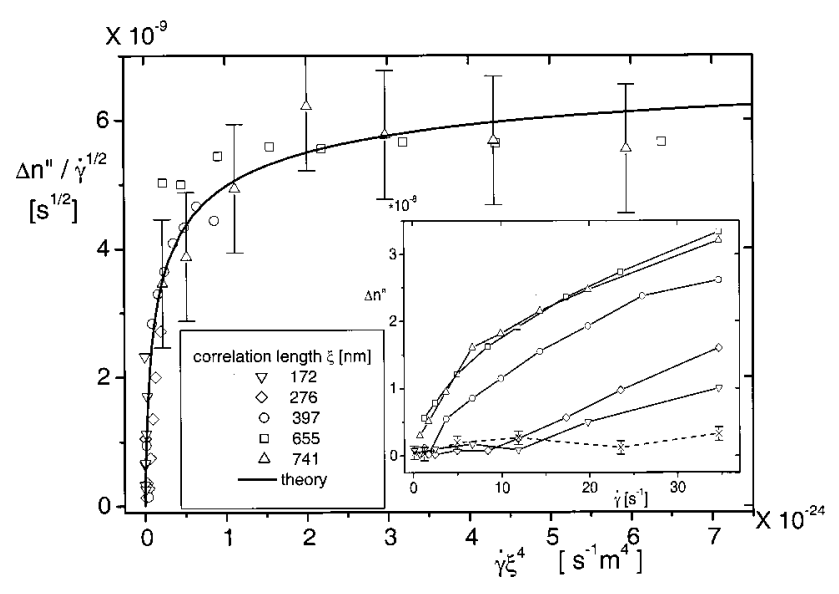

FIG. 11. Scaling of the experimental data. The solid line corresponds to the theoretical result [Eqs. (15) and (19)]. Inset shows dichroism measurements in the mean-field region. The dashed line is the dichroism for a sample without polymer.

against $\dot{\gamma} \xi^{4}$ all experimental curves should collapse onto each other. To within experimental errors, this is indeed seen to be the case in Fig. 11. In addition, the functional form of the dichroism scaling function Eq. (15), as depicted in Fig. 3, can be compared to the experimental master curve in Fig. 11 as follows. The dressed Peclet number $\lambda$ is directly proportional to $\dot{\gamma} \xi^{4}$. The proportionality constant between $\lambda$ and $\dot{\gamma} \xi^{4}$ can be found by rescaling the horizontal axis in Fig. 11, so as to obtain the best agreement between the theoretical scaling function and the experimental master curve. The vertical axis must be rescaled simultaneously. As can be seen from Fig. 11, the theoretical scaling function agrees with the experimental master curve to within experimental errors. The rescaling factor for the horizontal axis is equal to $\left(2 \beta \Sigma D_{0}\right)^{-1}$, according to Eq. (12), while the rescaling factor for the vertical axis is equal to $C_{\tau} \sqrt{R_{V}^{2} / 2 D_{0}} /\left(k_{0} R_{V}\right)^{4} k_{0}\left(\beta \Sigma / R_{V}\right)^{3 / 2}$, according to Eqs. (13) and (19). The scaling factors are found to be equal to 3.3 $\times 10^{2} \mathrm{sm}^{-4}$ and $2.0 \times 10^{-8} \mathrm{~s}^{1 / 2}$, respectively. From these numbers it follows that, $\beta \Sigma / R_{V}^{2} \approx 0.14$ and $\left(n_{p}-n_{f}\right)$ $\approx 0.025$, where $n_{p}$ and $n_{f}$ are the refractive index of the colloidal particles and the solvent, respectively. The former value is in accordance with a crude theoretical estimate $\left(\beta \Sigma / R_{v}^{2} \sim 0.1\right)[8,19]$, but differs by a factor of about ten with results obtained from earlier turbidity measurements on a similar colloidal system [20]. The refractive index difference of 0.025 is in good agreement with the value of 0.02 that is common for silica spheres prepared by the method of Stöber, in cyclohexane.

\section{Dichroism beyond mean field}

Dichroism as a function of the shear rate for correlation lengths larger than $750 \mathrm{~nm}$, corresponding to distances to the critical point that are beyond mean field, are plotted in Fig. 9 (filled symbols). Contrary to its mean-field behavior, shear induced dichroism decreases on approach of the critical point. In the mean-field region there is a bright streak in the scattering pattern, corresponding to the unaffected micro-
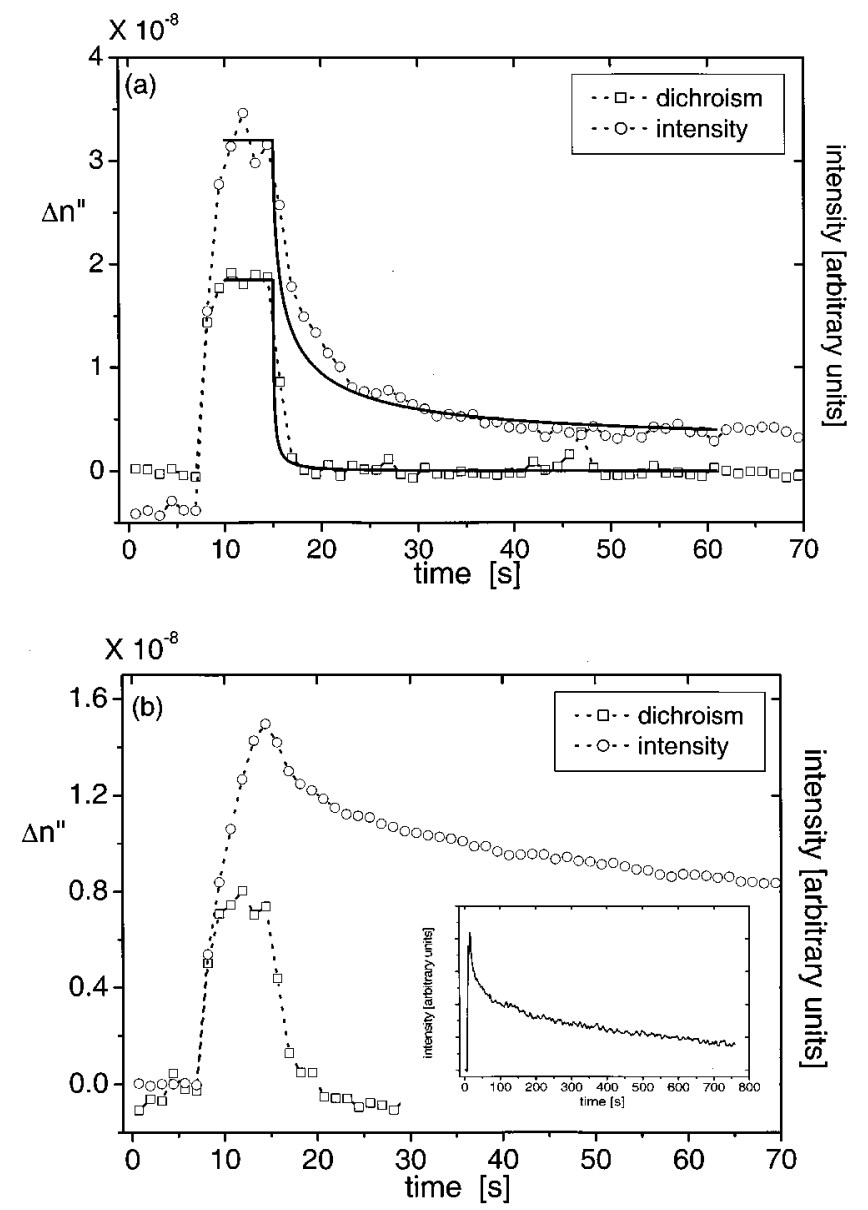

FIG. 12. (a) Relaxation curves for dichroism and the transmitted intensity for a correlation length of $600 \mathrm{~nm}$. The solid curves are theoretical mean-field predictions based on Eqs. (21) and (22). Shear flow is imposed at $7.5 \mathrm{~s}$ and is turned off at $15 \mathrm{~s}$. Plot (b) shows the relaxation of dichroism and transmitted intensity for the beyond mean-field region ( $\xi=1517 \mathrm{~nm}$ ). The inset in (b) shows the extremely long relaxation time for $\xi=1280 \mathrm{~nm}$.

structure in directions perpendicular to the flow direction [see remark just below Eq. (7) and Fig. 2]. What is observed beyond mean field is that the intensity of this bright streak is diminished when shear flow is applied, more so on closer approach of the critical point [21]. This breakdown of microstructure in directions perpendicular to the flow direction renders the structure factor more isotropic, leading to smaller dichroism. This effect of shear flow on the microstructure is lost when the equation of motion for the total-correlation function is linearized (as discussed in Sec. II C). Beyond the mean-field region one should consider nonlinear equations of motion. As far as we are aware, such nonlinear equations have not been analyzed yet.

\section{Relaxation of Dichroism and Turbidity}

Relaxation behavior was studied for correlation lengths of $600 \mathrm{~nm}$ (mean field) and $1517 \mathrm{~nm}$ (beyond mean field). Each measurement was started at time $t=0$ and then shear was applied at $t=7.5 \mathrm{~s}$, and turned off at $t=15 \mathrm{~s}$. Relaxation curves for dichroism and turbidity are given in Fig. 12. Fig- 
ure 12 (a) shows relaxation curves for a correlation length of $600 \mathrm{~nm}$, within the mean-field region. As can be seen, the shear induced dichroism relaxes within seconds, while the transmitted intensity (and hence, the turbidity) relaxes over a much larger time interval. As can be seen from Fig. 12(b), where relaxation curves for a correlation length of $1517 \mathrm{~nm}$ are plotted, beyond the mean-field region, relaxation of dichroism remains fast. Relaxation times of the turbidity, however, become larger on approach of the critical point. The insert in Fig. 12(b) illustrates the extremely long relaxation time of the turbidity on very close approach of the critical point $(\xi=1280 \mathrm{~nm})$. Hence, dichroism does not show critical slowing down, contrary to the turbidity. Note that the sample in the beyond mean-field region did not reach the stationary state at the time the shear rate is switched off.

These observed differences in relaxation behavior of dichroism and turbidity are in agreement with the mean-field theoretical predictions, as discussed in Sec. IIE [see Figure 12(a)]. The integrands in Eqs. (23) and (24) for dichroism are more sensitive to structure at larger wave vectors (because of the factor $K^{3}$ ) as compared to the integrand in Eq. (21) for the turbidity (a factor $K$ ). Since the dynamics of density waves with larger wave vectors is faster than for smaller wave vectors, this results in a faster relaxation of dichroism as compared to turbidity. The slower relaxation of the turbidity for larger correlation lengths is due to the smaller value of the effective diffusion coefficient [see Eq. (8)] for small wave vectors. The solid lines in Fig. 12(a) are theoretical relaxation curves, based on the equations in Sec. IIE. The dichroism relaxation curve is calculated from the constants derived from the scaling analysis of the stationary dichroism measurements in Sec. IV B 1, except that the constant $\left(2 \beta \Sigma D_{0}\right)^{-1}$ is chosen as $2.3 \times 10^{-8} \mathrm{~s}^{1 / 2}$ instead of 2.0 $\times 10^{-8} \mathrm{~s}^{1 / 2}$, in order to obtain agreement for the initial value of $\Delta n^{\prime \prime}$ (for a single dichroism measurement this lies within experimental errors). The theoretical intensity relaxation curve is obtained from the constants obtained in Sec. IV B 1, where the initial value of the intensity was an adjustable parameter (since we did not measure the incident intensity in this experiment) and the small experimental offset, due to optical imperfections of the shear cell, was added to the calculated intensity. The important thing to note here is that the theoretical curves reproduce, within experimental error, the very different experimentally observed relaxation times for dichroism and turbidity.

\section{SUMMARY AND CONCLUSION}

Shear induced dichroism near the gas-liquid critical point behaves completely different in the mean-field region and beyond mean field: for a given shear rate, dichroism first increases on approach of the critical point in the mean-field region, but then decreases in the beyond mean-field region. The decreasing dichroism on approach of the critical point for the beyond mean-field region is probably due to the breakdown of the structure factor in directions perpendicular to the flow direction. This phenomenon is due to nonlinear terms in the equation of motion for the structure factor. These nonlinear terms can be neglected in the mean-field region. So far, nonlinear equations of motion of the structure factor have not been analyzed. In the mean-field region, where equations of motion for the total-correlation function can be linearized, theory predicts scaling of data for $\Delta n^{\prime \prime} / \sqrt{\dot{\gamma}}$ when plotted against $\dot{\gamma} \xi^{4}$. This is confirmed by the experiments (see Fig. 11). Values for $\beta \Sigma / R_{V}^{2}$ and $n_{p}-n_{f}$, as found by comparing the experimentally obtained master curve and the theoretically predicted scaling function, are in accordance with a theoretical estimate for $\beta \Sigma / R_{V}^{2}[8,19]$ and independent experimental results for $n_{p}-n_{f}$ (see Sec. IV B 1). Shear rate dependent turbidity measurements close to the critical point on a similar colloidal system [20] rendered an unexpectedly high value of 3.6 for $\beta \Sigma / R_{V}^{2}$. The reason for this is not clear.

Relaxation experiments after cessation of the shear flow, show that dichroism does not exhibit experimentally relevant critical slowing down: relaxation times are found of the order of a second, right up to the critical point. This is in contrast to the turbidity, the relaxation time of which is found to diverge on approach of the critical point. This different dynamical behavior of dichroism and turbidity is in accordance with the mean-field theory. Formally, dichroism is more sensitive to the dynamics of density waves with larger wave vectors [due to the factor $K^{3}$ in the integrand in Eqs. (23) and (24)] as compared to the turbidity (a factor $K$ in the integrand in Eq. (21)). Since the dynamics of density waves are faster for larger wave vectors, this explains the relatively fast relaxation of dichroism. It is surprising however, that even very close to the critical point, the dichroism relaxation dynamics remains fast.

\section{ACKNOWLEDGMENTS}

We wish to thank Stichting voor Fundamenteel Onderzoek der Materie (F.O.M.) for financial support. We also wish to thank G. Harder, B. W. M. Kuipers, K. Rietveld, P. Engels, P. de Graaf, I. C. J. Dur, P. Bogerd, and M. C. A. de Groot for their contribution in the construction of the experimental setups.
[1] D. Beysens and R. Gastaud, Phys. Rev. A 30, 1145 (1984).

[2] D. Ronis, Phys. Rev. A 29, 1453 (1984).

[3] A. Onuki, J. Phys.: Condens. Matter 9, 6119 (1997).

[4] A. Onuki, K. Nakatani, and K. Kawasaki, Ann. Phys. (Leipzig) 131, 217 (1981).

[5] J. F. Scharwl and S. Hess, Phys. Rev. A 33, 4277 (1986).
[6] N. J. Wagner and W. B. Russel, Physica A 155, 475 (1989).

[7] J. K. G. Dhont, in An Introduction to Dynamics of Colloids, Studies in Interface Science, edited by D. Mobius and R. Miller (Elsevier, New York, 1996), Vol. II, p. 642.

[8] J. K. G. Dhont and V. Verduin, J. Chem. Phys. 101, 6193 (1994). 
[9] J. K. G. Dhont and H. Verduin, Physica A 235, 87 (1997).

[10] S. Asakura and F. Oosawa, J. Chem. Phys. 22, 1255 (1954).

[11] S. Asakura and F. Oosawa, J. Polym. Sci. 33, 183 (1958).

[12] A. Vrij, Pure Appl. Chem. 48, 471 (1976).

[13] H. N. W. Lekkerkerker, W. C. K. , P. N. Pusey, A. Stroobants, and P. B. Warren, Europhys. Lett. 20, 559 (1992).

[14] V. G. Puglielli and N. C. Ford, Jr., Phys. Rev. Lett. 25, 143 (1970).

[15] J. K. G. Dhont, Phys. Rev. Lett. 76, 4269 (1996).
[16] W. Stöber, A. Fink, and E. Bohn, J. Colloid Interface Sci. 26, 62 (1968).

[17] G. G. Fuller, in Optical Rheometry of Complex Fluids, 1st ed., edited by K. E. Gubbins (Oxford University, New York, 1995), p. 268.

[18] G. G. Fuller and K. J. Mikkelsen, J. Rheol. 33, 761 (1989).

[19] M. Fixman, Adv. Chem. Phys. 6, 175 (1964).

[20] H. Verduin and J. K. G. Dhont, Phys. Rev. E 52, 1811 (1995).

[21] J. K. G. Dhont and I. Bodnár, Phys. Rev. E 58, 4783 (1998). 